\title{
Vorwärts zu den Anfängen
}

n Heft 2/97, S. 73, beklagte Professor Borelli eine mangelhafte Beachtung dermatologischer Meriten in der Entwicklung des Faches Allergologie („Der Dermatologie innerhalb der Geschichte der Allergologie stärker Rechnung tragen"). Folgt man seiner Begründung - mit Beispielen dermatologischer Beiträge zum Erkenntnisfortschritt des Faches -, so könnten eine Vielzahl von Disziplinen in die Klage einstimmen: Innere Medizin, Pneumologie, HNO-Heilkunde, Pädiatrie u.a.m. Es liegt in der Natur des interdisziplinären Faches Allergologie, daß je nach Dynamik der beteiligten Fachrichtungen bzw. dem Auftreten herausragender Wissenschaftler das Gewicht der Disziplinen mit den Zeitläuften variiert. Und dies ist auch gut so, beflügeln doch gerade die aktiven Gruppen, unabhängig von deren Ausrichtung, die allergologische Weiterentwicklung (,Konkurrenz belebt das Geschäft"“).

Die Entwicklung des Faches Allergologie ist keineswegs zum Abschluß gekommen. Auf dem Wege nach Europa werden neue Modelle diskutiert, vor allem der „Facharzt für Allergologie und klinische Immunologie“. Wissenschaftliche Impulse könnten von einer derartigen Fachrichtung sicherlich ausgehen. Erhebliche Nachteile wären jedoch $\mathrm{zu}$ erwarten für die praktische Versorgung allergischer Patienten, vor allem aber auch für den praktizierenden „Organmediziner“. Man stelle sich den allergologisch erfahrenen Dermatologen, Pädiater, Pneumologen oder HNO-Arzt vor, der „seinen“ Ekzematiker, Rhinitiker, Asthmatiker zur Allergiediagnostik an einen „Allergologen“ neuer Prägung überweisen müßte, um einen Hauttest bzw. Provokationstest durchführen zu lassen. Dahingestellt sei, ob der „Allergologe“ bereit sein würde, „seinen“ „Allergiker" dem Organspezialisten zur Differentialdiagnose zu überweisen.

Eine derartige Aufsplitterung der Kompetenz kann und darf sich nicht durchsetzen. Dies ist zumindest die Auffassung der DGAI - an den Kommentaren der Leser zu dieser Thematik wären die Herausgeber sehr interessiert. Die DGAI folgt zur Zeit einem Vorstandsbeschluß, der auch weiterhin die Allergologie als Ergänzung zu einer soliden Weiterbildung in einem „organbezogenen“ Fach befürwortet. Offen ist aus Sicht der DGAI, wie in Fächern wie Innere Medizin, Dermatologie, Pädiatrie oder HNOHeilkunde die notwendige Kompetenz in allen Weiterbildungsstellen erworben werden soll. Unabhängig davon dürfte zur Förderung der Wissenschaft die Einrichtung einiger „Allergiezentren“ sehr dienlich sein, wie sie zur Zeit vom BMBF gefördert werden soll.

Zurück zu Borelli: Welche Fächer standen bei der Erschaffung der Allergologie Pate? Die DGAI beispielsweise wurde 1951 gegründet von Hygienikern (5), Internisten (4), Pathologen (2), Pharmakologen (1), Augenärzten (1), wobei zu konstatieren ist, daß sich Fächer wie die Immunologie erst später abgegrenzt haben, u.a. von der Hygiene.

Der erste experimentelle Allergologe in heutigem Sinne war $\mathrm{Ch}$. $\mathrm{H}$. Blackley (1820-1900), ein Homöopath. Der praktische Arzt Heberden (1710-1801) beschrieb 1802 das Pollenasthma, wie in Deutschland später der Pharmakologe Phoebus (1804-1880). Der Hygieniker Dunbar (1863-1922) in Hamburg verhalf der Pollenätiologie des Heuschnupfens zum Durchbruch.

Somit haben zahlreiche Disziplinen zur Entwicklung des Faches Allergologie beigetragen, wobei die Wissenschaftler oft mehr als durch ihre Fachdisziplin durch ihre eigenen allergischen Erkrankungen motiviert waren.

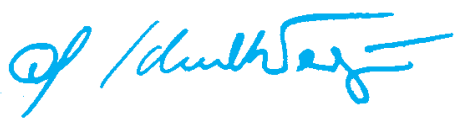

Prof. Dr. G. Schultze-Werninghaus
Bekanntes Beispiel hierfür ist - neben den o.g. Wissenschaftlern - auch A. Trousseau (1801-1867), der sich um die Weiterentwicklung von Diagnostik und Behandlung des Asthma bronchiale verdient gemacht hat.

Eine „Aufrechnung“ der an der allergologischen Entwicklung beteiligten Fächer bezüglich ihrer Beiträge ist nicht nur wenig hilfreich, sondern auch deshalb unsinnig, da sich eine Reihe von heutigen Disziplinen, wie die Immunologie, erst im Verlauf der Zeit abgegrenzt hat.

Vorwärts kann sich das Fach Allergologie nur dann entwickeln, wenn wir uns rückbesinnen auf die multidisziplinären Anfänge, in denen die Motivation des einzelnen Arztes das wichtigste Moment der Entwicklung war. Statt Streit um Anteile einzelner Disziplinen sollte die Allergologie geprägt werden durch das Engagement qualifizierter Kollegen, die ihre Disziplin ergänzen wollen durch eine Spezialausbildung in Allergologie. Das Fach Allergologie lebt von der Spezialisierung im Rahmen einer festen Verankerung in der organbezogenen Weiterbildung, sowohl bei Hautkrankheiten als auch bei Erkrankungen der oberen und tieferen Atemwege.

Das Fach lebt auch von der ständigen Begegnung der beteiligten Disziplinen. Daher sei auch an dieser Stelle an alle Allergologen in Grundlagenforschung, Klinik und Praxis appelliert, einer Aufsplitterung in eigene Organisationsstrukturen, Tagungen und Fachorgane für jede allergologische Subdisziplin entgegenzuwirken, mit der Gefahr, daß eines Tages die kritische Masse nicht mehr ausreicht, um das Fach Allergologie in Wissenschaft und Politik erfolgreich zu vertreten. Auch hier sollten wir uns auf die Anfänge besinnen!

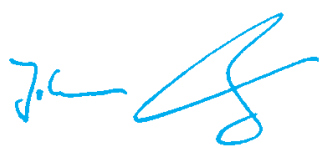

Prof. Dr. Dr. J. Ring 